\title{
ADAPTIVE ALTERNATIVE PATH AND RATE BASED CONGESTION CONTROL FOR 6LOWPAN, WSN TOWARDS INTERNET OF THINGS
}

\author{
RAMADEVI CHAPPALA * \\ Research Scholar, Department of CSE, Acharya Nagarjuna University, Guntur, India. \\ E-mail: ramadevisibbala2007@gmail.com \\ CH.ANURADHA \\ Assistant Professor, Department of CSE, \\ VRK Siddhartha Engineering College, Vijayawada, Andhra Pradesh, India \\ P.SRI RAM CHANDRA MURTHY \\ Assistant Professor, Department of CSE, Acharya Nagarjuna University, Guntur, India
}

\begin{abstract}
Internet of Things (IoT) is a common Global platform to connect various heterogeneous devices over Internet which enable Machine-to-Machine (M2M) communication by embedding intelligent software in the objects to deploy high-quality and effective smart devices to offer different types of services. Congestion will be increased with the rate of increasing devices in the IoT networks. In congestion control mechanisms based on rate adjustment, dropping a packet will cause entire packet re-transmission due to packets are usually fragmented. In case of an alternative path approach, finding an optimal path with minimum hop count, low buffer occupancy, maximum link quality, low packet dropping rate and maximum through put is not easy to establish in every circumstance. In this paper, we propose an Adaptive Hybrid Congestion Control Protocol (AHCCP) for RPL based IoT sensor networks. In this protocol, initially the packets are sensed by an end IoT devices are categorized into various priorities depending on traffic type. Based on congestion status source will choose either rate based or alternative path for data transmission. This protocol will be implemented in NS3 and compare the results with game theory based congestion control protocol in terms of PDR, throughput, packet loss and energy efficiency.
\end{abstract}

Keywords: AHCCP, Progressive Filling, Internet of Things, Game Theory, Traffic Classification

\section{Introduction}

Internet of Things gaining huge research attention due to the requirement of different type of network integration. It is a future of Internet also considered as Internet of Objects (IoO), Internet of Environment (IoE) and Machineto-Machine (M2M) communication. For smart objet communication IoT has been considered as a leading technology in today's world. It is an intelligent combination of sensor devices, processing unit which can be placed on cloud with uniquely identifiable objects, communication infrastructure, action invoking and decision making system. Different priorities can be assigned to these smart sensor devices. The research highlights, there are smart devices which can be beyond the people on earth in 2008, 16 billions of smart devices in 2016 and this number can be increased up to 29 billion in 2022[1]. Day by day the devices and applications corresponding to IoT network are increased rapidly. Applications covers from [2] IoT are healthcare monitoring system, smart grid, smart homes, smart cities, traffic and shipping of goods, ITS [3] and predicting natural disasters. IoT is not a single technology to support these various applications. Instead several complementary technical developments which provide capabilities of communication, co-operation, addressability, Identification, sensing, actuation, localization, information processing decision making and user interface are taken together to bridge the gap between virtual and physical world. However, majority of such connected devices are constrained with limited processing power, memory, speed and buffer occupancy and energy constraints. Therefore, it is essential to make standard protocols that enable these technologies including ubiquitous and pervasive computing, communicating technologies and embedded devices. Different types of application protocols supported by IoT are MQTT, XMPP and RESTful HTTP to provide data communication over the Internet. CoAP protocol is a web-based, light weight RESTful application layer protocol to define the basic congestion control for IoT applications which operates on top of UDP [4]. Due to the extensive features of IPV6 it support for stateless address auto configuration, popularization, easy to access, applicability and sufficient address space. We can easily connect and access these features of smart devices which are conforming to the standard IEEE 802.15.4. IETF introduce an adaptive layer in devices with IEEE 802.15.4 is IPv6 over Low Power Wireless Personal Area Network [5]. With this 
implementation IEEE 802.15.4 had an ability to forward, process and receive IPv6 (transmission data size is 1280 bytes) packets though the transmission size differ from IEEE802.15.4 (transmission unit size is 127 bytes). IETF standardized IPv6 Routing Protocol for Low Power and Lossy networks is a tree topology. In this topology if an event occurs at leaf node, it is very difficult to handle congestion because all nodes in that region causes congestion by sending packets toward sink node. Basically, RPL protocol uses parent-change mechanism to select a parent with less hop count, minimum transmission count and with an excellent link quality so that it can avoid repeated change of state from one to another called ping-pong effect in which the parent changes frequently which leads to packet loss, increasing packet delay and decreasing through put [6]. Basically congestion control protocols are divided into traffic control and resource control based protocols. In resource control congestion mitigation depends on the type of resources distributed among smart devices in an IoT network. Traffic control mechanisms are classified into rate adjustment where the nodes will decrease their sending rate to reduce the number of packets in global and local networks and an alternative path scheme select an optimal path to transmit packet when congestion detect in the network.

In our protocol we use both types of traffic control mechanisms by eliminating drawbacks of rate-based approach. And we compare our results with the existing Game Theory Based Congestion Control protocol to maximize throughput and energy efficiency levels, reduce packet loss, E-2-E delay and also control the overhead involved in data transmission in RPL based Low Power Lossy Networks (LLN's).

\section{Organization of paper}

The remaining part of the paper is organized as follows.

Section 2 describes the related work. Section 3 presents the proposed work based on three different methodologies. In Section 4 the experimentation carried out and corresponding results are mentioned. Finally conclusion is discussed.

\section{Related work}

Congestion is the major problem occurs in almost all wired, wireless and hybrid networks. IoT is a combination of heterogeneous networks with constrained devices having low power, low speed, low processing and storage area. There are different protocols, algorithms and mechanisms for congestion control in IoT sensor networks. In our work we focus on three types of traffic control methods to avoid congestion in RPL based LLN's. Finally, the compactness of the congestion is supported by packet service time, back pressure, priority based and learning based approach. Also the channel free and channel state transmission are the important issues to be considered for the compactness of congestion control methods.

\subsection{Congestion control mechanism based on rate-based approach}

The basic simple COAP-CC imposes maximum limit on outgoing message of rate of transmission using exponential back-off between retransmission of lost messages [7] and concurrent messages exchanged between source node and sink node using NSTART value placed in COAP specification. The author in [8], proposed $\mathrm{CoCoA}^{+}$to control the rate of transmission it uses the three functions. First, depending on the network conditions RTO is calculated. Second, RTO value is modified by a back off method for retransmission. Finally, gets the network status information using Aging policy.

In [9], the author uses packet service time as the congestion metric in Priority based Congestion Control protocol (PCCP). The source node makes use of upward node transmission rate and packet priority to set its transmission rate. The authors proposed Congestion Detection and Avoidance (CODA) which uses wireless channel load and queue length as congestion metric [10]. When source does not receive ACK, it reduces the rate of transmission. CONSEQ is the hop-by-hop congestion control and load balancing scheme proposed in [11], where it uses Effective Queue Length (EQL) as congestion metric. Fuzzy logic is also used in this method when congestion is not mitigated. In [12], the author propose a protocol uses both traffic control and resource control strategy called an Optimization based Hybrid Congestion Control method uses multi-attribute optimization methodology to forward packets in non-congested path to improve network throughput and lifetime. The author propose DDR [13] in which the buffer occupancy is more than maximum threshold then queuing theory is used to set new transmission rate also measure least hop count and assign it to the node which are close to the sink node.

\subsection{Congestion control based on alternative path approach}

The author proposed new approach Siphon [14], distributes a special virtual sink nodes having powerful radio than ordinary sensor nodes in the whole network. Initially, the virtual sink receives a packet from source node and it forward to physical real sink via another radio networks like WiFi, Zigbee etc., but it is infeasible to implement in constrained environment. Another protocol [15] Location Aided Congestion Aware Routing mechanism makes use of average number of successful transmission attempts and buffer occupancy to detect congestion. LACAR uses each neighbor and sink node to provide an optimal path with less energy consumption and less congestion along with other congestion metrics. Another protocol Traffic Aware Dynamic Routing (TADR) treats the whole network traffic pattern as a bowl and all data packets going down towards sink which is residing at the bottom. 
Queue length potential force and depth field force used to find out the next neighbor to forward packet [16]. The authors proposed coalition game theory assign Cell Access Nodes (CANs) to achieve Nash-Equilibrium. In [17] the author proposes an optimal congestion control for cross layer networks to control at link level and node level in WSNs. These algorithms ensure maximum throughput, efficient energy levels, effective data transmission and low communication overhead.

\section{Proposed Work}

The proposed solution includes an Adaptive Hybrid Alternative path Congestion Control mechanism to mitigate congestion in RPL tree topology for low power, Lossy networks. This protocol has mainly three stages.

\subsection{Priority Assignment for data traffic in IoT networks}

Every packet is sensed by an end IoT devices are classified into various priorities. Also different types of devices consist of different priorities in IoT networks. The data traffic that need QoS like fire alarming, medical sensors, seismic sensors classified under high priority and other traffic considered as low priority data. Table1: shows four types of traffic classes. And in this, lower value represents higher priority.

Table 1. Traffic Classification

\begin{tabular}{|l|c|}
\hline \multicolumn{1}{|c|}{ Traffic Class } & Traffic class Priority \\
\hline Real-time reliable (RR) & 1 \\
\hline Real-time Non-reliable (RnR) & 2 \\
\hline Non-Real time Reliable (nRR) & 3 \\
\hline Best Effort Traffic & 4 \\
\hline
\end{tabular}

The brief description of traffic classes are as follows

RR traffic is both delay and reliability-constrained. It corresponds to critical data packets that need to reach the sink with high reliability and within a stringent delay-deadline.

$R n R$ Traffic, also known as delay-constrained packets, must reach the sink within a strict delay-deadline. However, some packet losses may be tolerated. This type of traffic may carry, for example, multimedia data and video streaming.

$n R R$ Traffic is highly reliability constrained but not delay-constrained.

$B E$ Traffic is neither delay-constrained nor reliability-constrained. They are also known as normal packets and only require best effort support.

This mechanism depends on two conditions- First, the network performance is good enough when $\operatorname{Avg}_{\text {loss rate }}>$ Recent loss rate. Second, we consider the network performance is worst when $\mathrm{Avg}_{\text {loss rate }}<$ Recent loss rate.

Based on the above two conditions RTO value and Back off Timer is adjusted.

\subsection{Congestion Control based on Optimal Adaptive Alternative path Mechanism}

Once the traffic flow is scattered with priority then Optimal Adaptive Alternative path based mechanism choose best optimal path with sufficient band width, maximum link quality, minimum buffer occupancy and least hop count in a way to maximize throughput, energy efficiency levels and minimize packet drop rate. When congestion is detected by a child node with the net packet flow rate $\left(\alpha=\mathrm{r}_{\text {gen }}-\mathrm{r}_{\text {out }}\right)$ as congestion metric in RPL topology, then the child node invoke set of procedures based on Game Theory to find an appropriate parent. Each node distribute DIO packet to local neighbors periodically to keep network connectivity. DIO packet contains the information about unique identity of network, sender's rank field and an optional field used to calculate expected rank of a node. If this expected rank is less than present rank then the node will invoke a parent change procedure based on channel status to minimize its rank value. Several metrics to calculate rank of a node according to "Eq. (1) are throughput, expected rank of a node, energy state and hop count.

$$
\operatorname{Rank}(\mathrm{i})=\mathrm{RI}+\mathrm{LQp}+\operatorname{Rankp}
$$

Where RI is the rank increasing value, LQ is the link quality and Rank $k_{\mathrm{p}}$ is the rank of a parent. When congestion is detected $\mathrm{CN}$ bit is set in DIO packet and invoke parent change procedure.

\subsection{Adaptive Hybrid Rate Controlled Approach}

When congestion is not mitigated once we use the above two methods, the congested parent invoke rate controlled algorithm to reduce rate of transmission. Flow control and congestion control are most widely used algorithm in traditional networks. Adaptive Rate controlled mechanism determines the network link capacity and bandwidth to estimate the exact packet sending rate of a source node. In RPL tree topology the sink node invoke rate controlled mechanism by considering each source node request for an equal part of bandwidth and use progressive filling algorithm to calculate reasonable min-max fair packet rate allocation. 


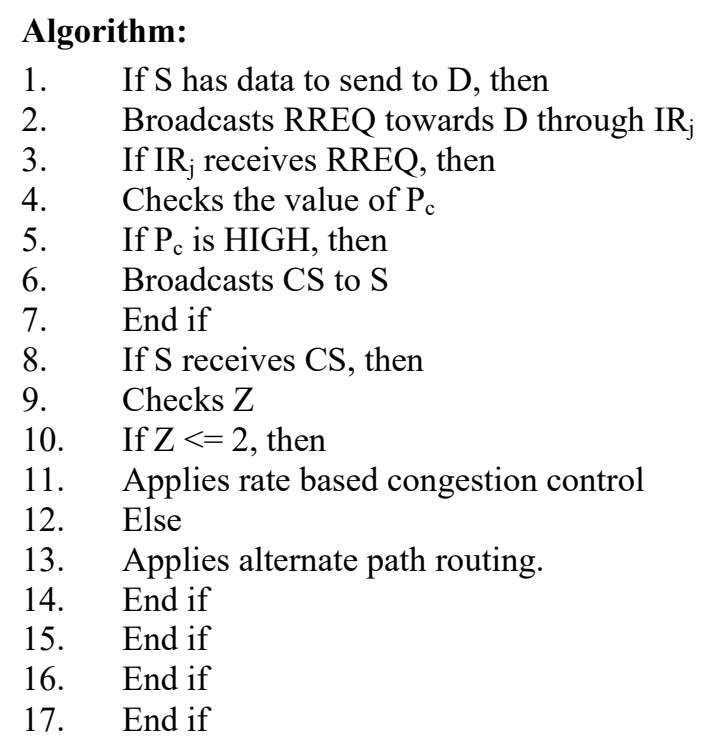

Table 2. Notations Used in Algorithm

\begin{tabular}{|l|l|}
\hline Notations & \multicolumn{1}{c|}{ Definition } \\
\hline $\mathrm{X}$ & Congestion status \\
\hline $\mathrm{P}_{\mathrm{C}}$ & Congestion probability \\
\hline $\mathrm{S}$ and $\mathrm{D}$ & Source and destination (sink) \\
\hline $\mathrm{IR}_{\mathrm{j}}$ & Intermediate routers, $\mathrm{j}=1,2 . . \mathrm{m}$ \\
\hline $\mathrm{CS}$ & Congestion status \\
\hline $\mathrm{Z}$ & Number of routers forwarding CS message \\
\hline RREQ & Route Request packet \\
\hline
\end{tabular}

\section{Simulation Results}

\subsection{Simulation Parameters}

We use NS3 to simulate our proposed Adaptive Hybrid Congestion Control Protocol (AHCCP) IoT Sensor Networks. We use the IEEE 802.11 for wireless sensor networks as the MAC layer protocol. It has the functionality to notify the network layer about link breakage. In our simulation, the number of nodes is varied as $21,41,61,81$ and 101 . The area size is 50 meter $\mathrm{x} 50$ meter square region for 50 seconds simulation time. The simulated traffic is Exponential. Our simulation settings and parameters are summarized in table 3.

Table 3. Simulation Parameters

\begin{tabular}{|l|l|}
\hline Parameter & Description \\
\hline Number of nodes & 31 \\
\hline Size of topology & 1500 X 300 \\
\hline MAC & 802.11 \\
\hline Simulation Time & $50 \mathrm{sec}$ \\
\hline Traffic Source & Exponential \\
\hline Number of Flows & $2,4,6,8$ and 10 \\
\hline Propagation Type & Two Ray Ground \\
\hline Antenna Model & Omni Antenna \\
\hline Initial Energy & 10.1 Joules \\
\hline Transmission Power & 0.3 watts \\
\hline Receiving Power & 0.3 watts \\
\hline Transmission Rate & $50,75,100,125$ and $150 \mathrm{~Kb}$ \\
\hline
\end{tabular}




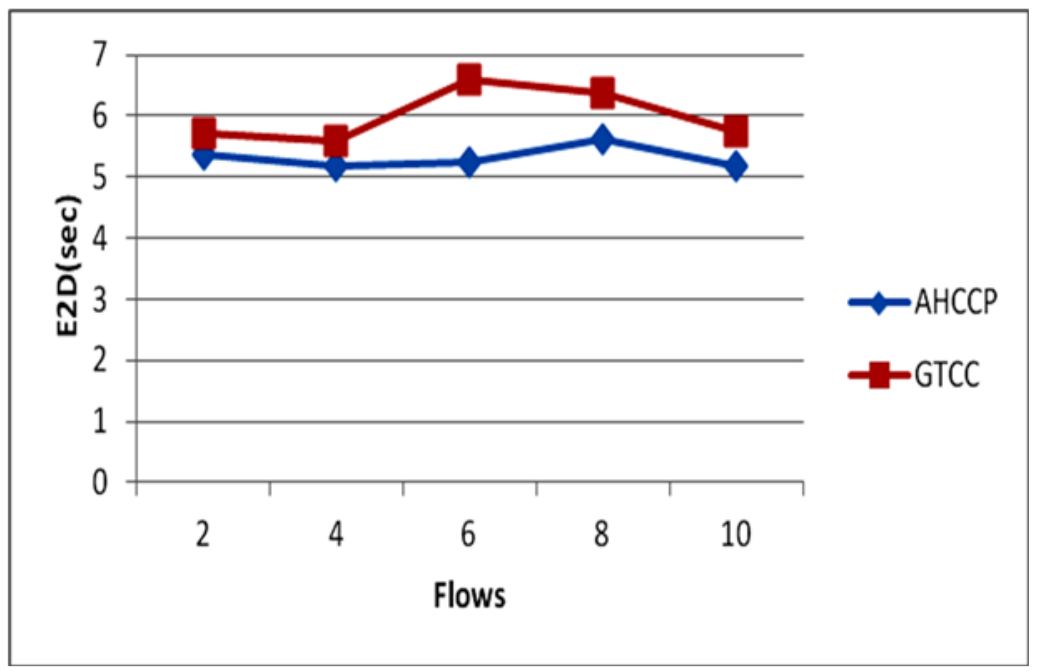

Fig. 1 End-to-End Delay for varying Flows

The graph showing the results of E2D for varying the flows is shown in Fig. 1. The figure depicts that the E2D of AHCCP ranges from 5.3 to 5.1 seconds and E2D of GTCC ranges from 5.72 to 5.74 seconds. Ultimately, the E2D of AHCCP is $11 \%$ less when compared to GTCC

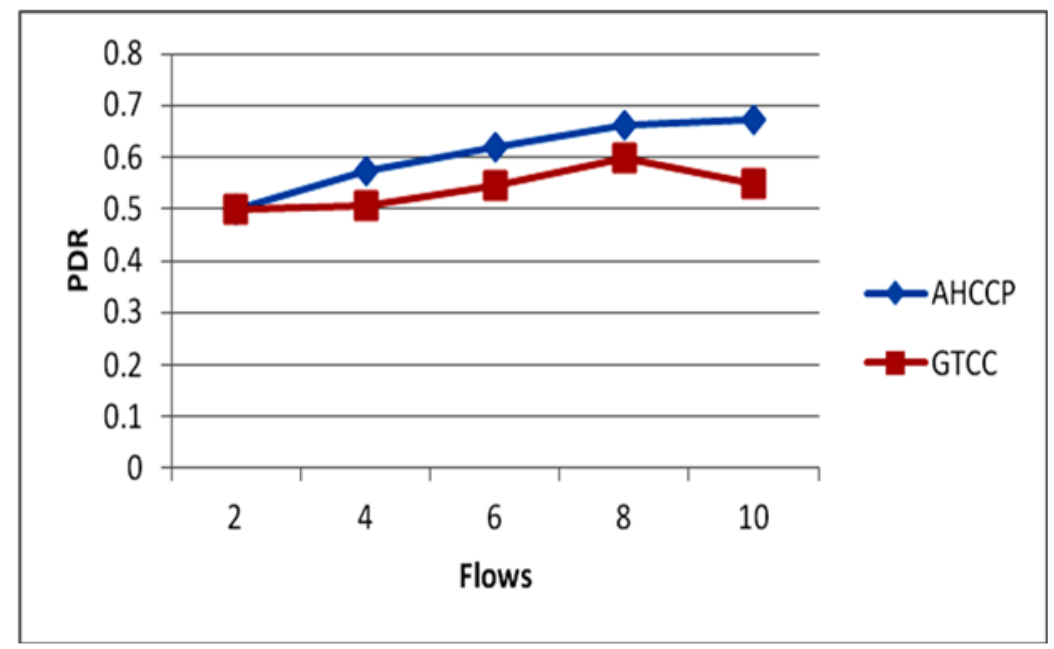

Fig. 2 PDR for varying Flows

The graph showing the results of PDR for varying the flows is shown in Fig. 2. The figure depicts that the PDR of AHCCP ranges from 0.49 to 0.67 and PDR of GTCC ranges from 5.72 to 5.74. Ultimately, the PDR of AHCCP is $10 \%$ high when compared to GTCC

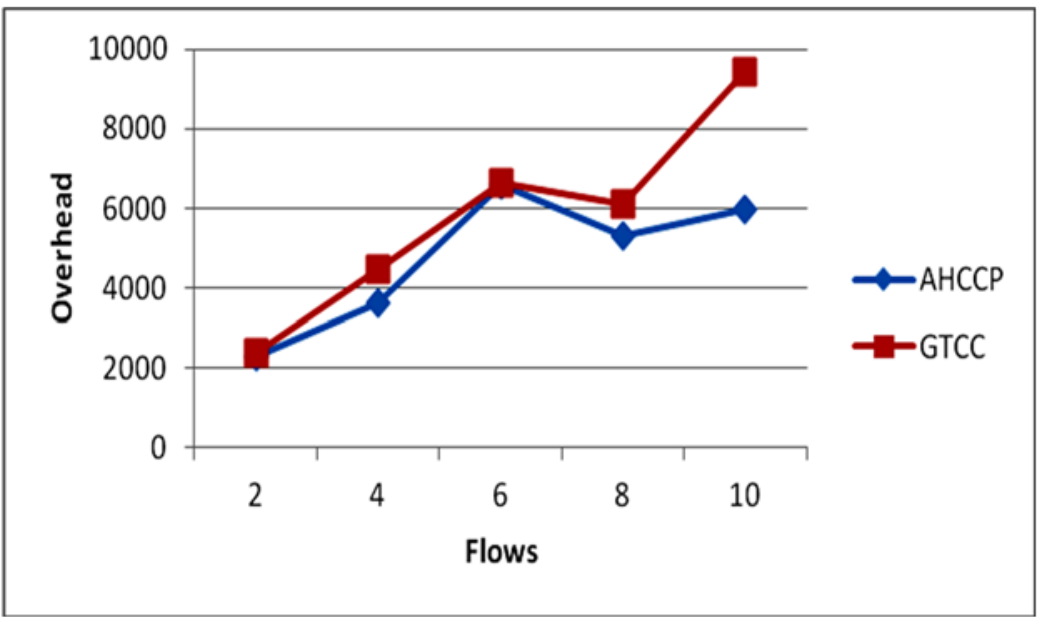

Fig. 3 over head for varying Flows 
The graph showing the results of overhead for varying the flows is shown in Fig.3. The figure depicts that the overhead of AHCCP ranges from 2292 to 5992 and overhead of GTCC ranges from 2368 to 9442 . Ultimately, the overhead of AHCCP is $14 \%$ less when compared to GTCC

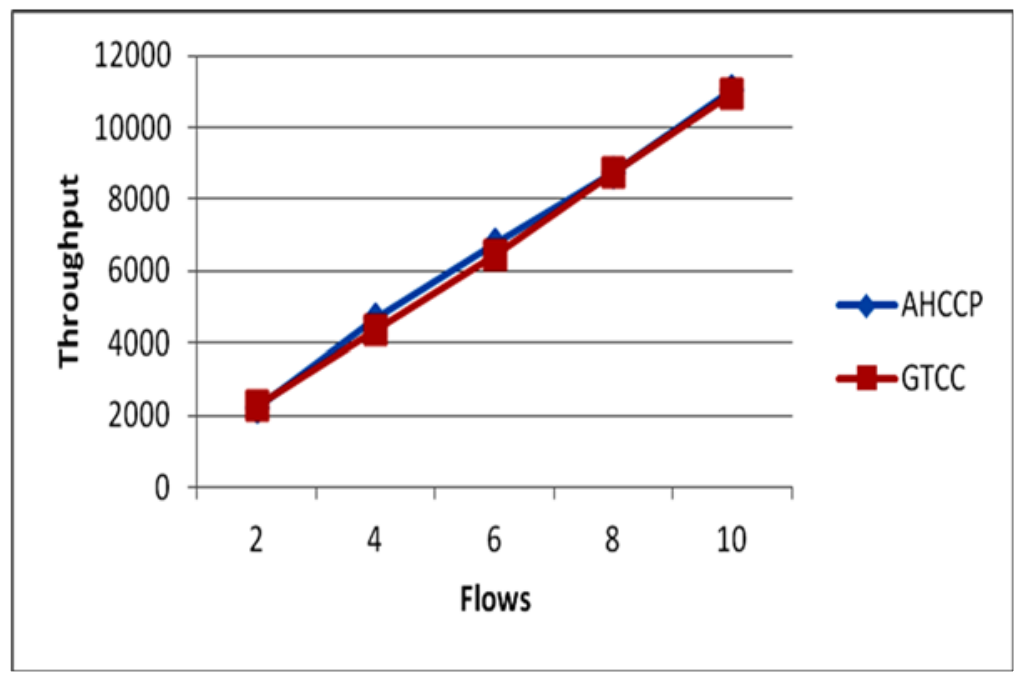

Fig. 4 Throughput for varying Flows

The graph showing the results of throughput for varying the flows is shown in Fig. 4. The figure depicts that the throughput of AHCCP ranges from 2231 to 11056 and throughput of GTCC ranges from 2276 to 10931. Ultimately, the throughput of AHCCP is $2 \%$ high when compared to GTCC.

\subsection{Based on rate of control}

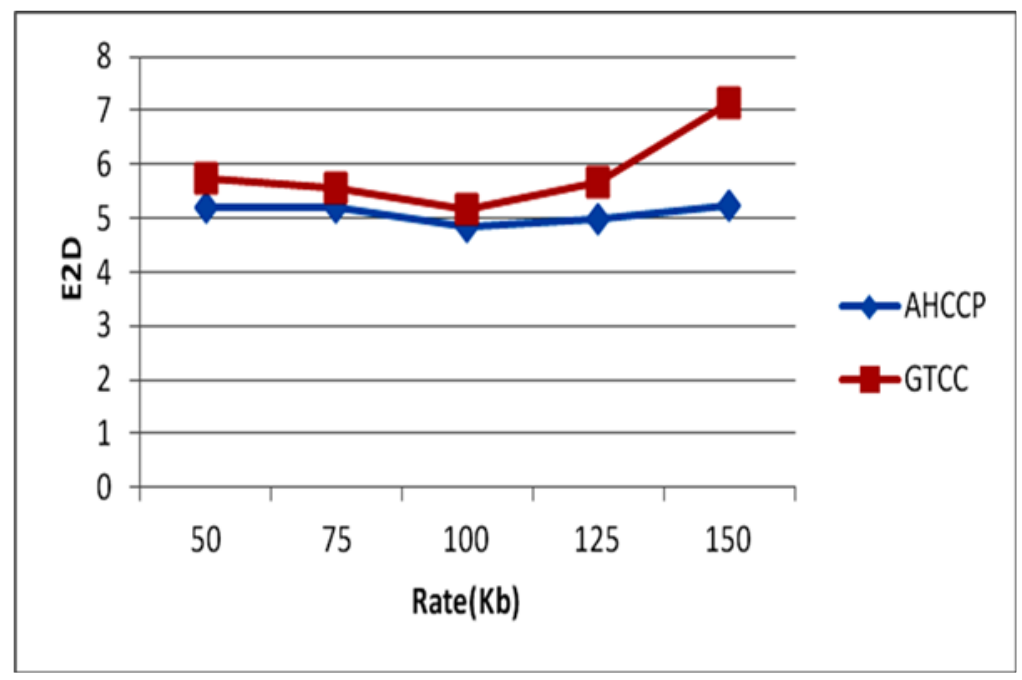

Fig. 5 End-to-End Delay for varying Rate

The graph showing the results of E2D for varying the rate is shown in Fig. 5. The figure depicts that the E2D of AHCCP ranges from 5.1 to 5.2seconds and E2D of GTCC ranges from 5.7 to 7.1 seconds. Ultimately, the E2D of AHCCP is $12 \%$ less when compared to GTCC 


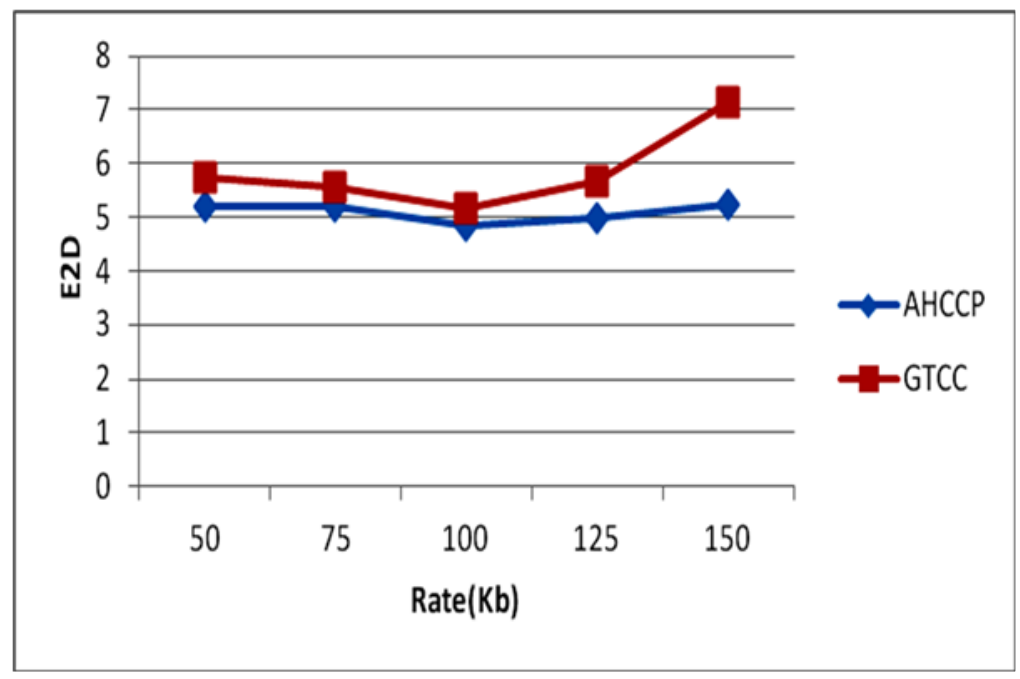

Fig. 6 PDR for varying Rate

The graph showing the results of PDR for varying the rate is shown in Fig. 6. The figure depicts that the PDR of AHCCP ranges from 0.67 to 0.61 and PDR of GTCC ranges from 0.54 to 0.53 . Ultimately, the PDR of AHCCP is $10 \%$ high when compared to GTCC

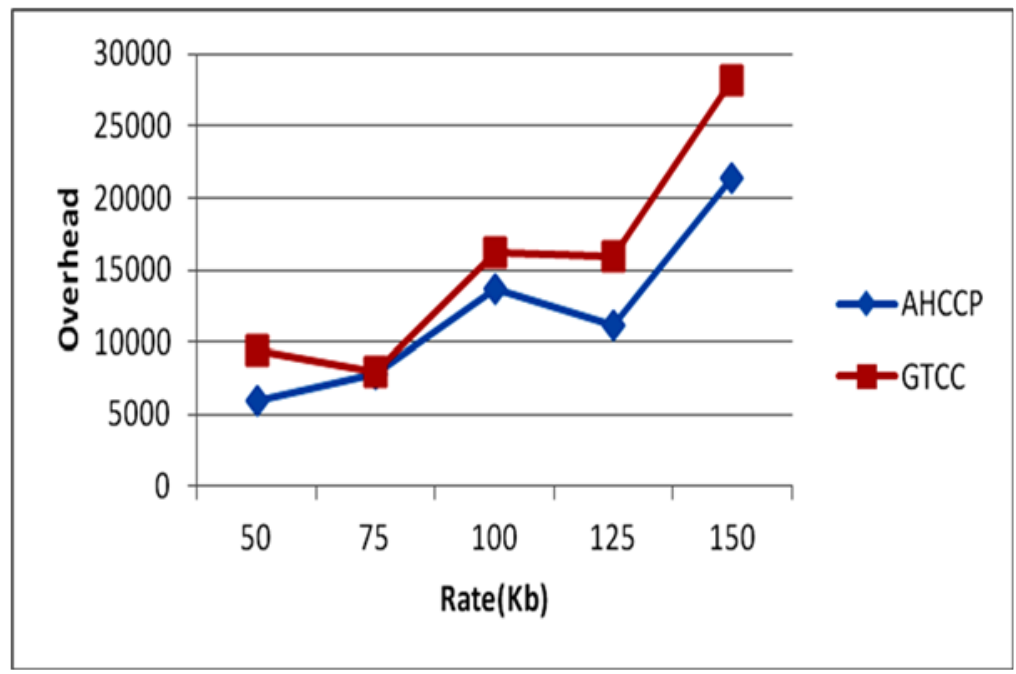

Fig. 7 Overhead for varying Rate

The graph showing the results of overhead for varying the rate is shown in Fig. 7. The figure depicts that the compared to overhead of AHCCP ranges from 5992 to 21407 and overhead of GTCC ranges from 9442 to 28222. Ultimately, the overhead of AHCCP is $22 \%$ less when GTCC 


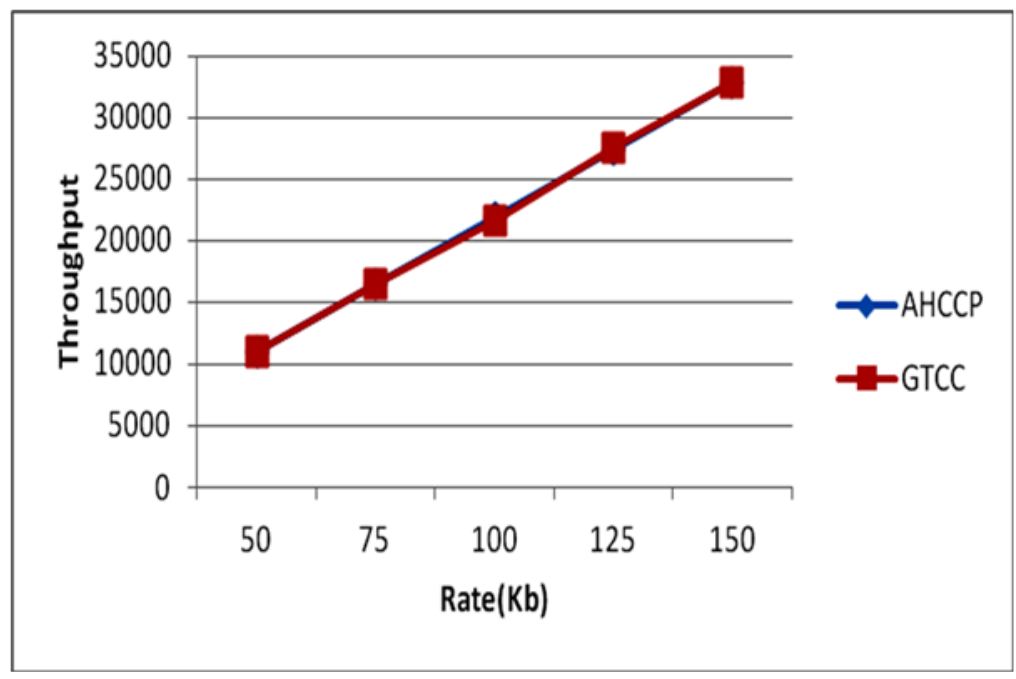

Fig. 8 Throughput for varying Rate

The graph showing the results of throughput for varying the rate is shown in Fig. 8. The figure depicts that the throughput of AHCCP ranges from 11056 to 32892 and throughput of GTCC ranges from 10931 to 32797. Ultimately, the throughput of AHCCP is $0.44 \%$ high when compared to GTCC.

\section{Conclusion}

In this paper, we have proposed an adaptive hybrid congestion control protocol for IoT sensor network. In this protocol, the packets sensed by the end Internet of Things devices are categorized into various priorities, depending on the traffic type. Based on the congestion status, source will use either rate based congestion control technique or choose alternate path for data transmission. By simulation results, we have shown that the proposed protocol is implemented in NS3 and compared with existing approaches in terms of packet delivery ratio, packet drop, through put and energy efficiency

\section{References}

[1] Ericsson mobility report June 2017 https://www.ericsson.com/assets /local /mobilityreport /documents /2017/ericsson-mobility-reportjune-2017.pdf.

[2] D. Bandyopadhyay, J. Sen, (2011) “Internet of things: applications and challenges in technology and standardization". Wireless Personal Communication, 58 (1):49-69.

[3] J. Zheng, D. Simplot-Ryl, C. Bisdikian, \& H. Mouftah, "The Internet of Things," in IEEE Communications Magazine, Volume: 49, Issue: 11, pp: 30-31, 2011.

[4] C. Bormann, A. P. Castellani, and Z. Shelby, "CoAP: An Application Protocol for Billions of Tiny Internet Nodes," IEEE Internet Comp., vol. 16, no. 2,Mar. 2012, pp. 62-67.

[5] J. Gubbi, R. Buyya, S. Marusic, M. Palaniswami, "Internet of Things (IoT): A vision, Architectural Elements, and Future Directions," Future Generation Computer Systems, Vol. 29, pp. 1645-1660, 2013.

[6] T. Winter, P. Thubert, A. Brandt, J. Hui, R. Kelsey, P. Levis, K. Pister, R. Struik, J. Vasseur, and R. Alexander, "RPL: IPv6 Routing Protocol for Low-Power and Lossy Networks (RPL)", RFC 6550 (Proposed Standard), Internet Engineering Task Force,March2012.

[7] Z. Shelby, K. Hartke, and C. Bormann, "The Constrained Application Protocol (CoAP)," IETF RFC 7252, June 2014.

[8] R. Bhalerao, S. S. Subramanian, and J. Pasquale, "An analysis and improvement of congestion control in the coap internet-of-things protocol," in Consumer Communications \& Networking Conference (CCNC), 2016 13th IEEE Annual. IEEE, 2016, pp. 889-894.

[9] N. Tsiftes, J. Eriksson, and A. Dunkels, "Poster abstract: low power wireless IPv6 routing with ContikiRPL," in Proceedings of the 9th ACM/IEEE International Conference on Information Processing in Sensor Networks, pp. 406-407, Stockholm, Sweden, April 2010.

[10] C.Y. Wan, S. B. Eisenman, and A. T. Campbell, "Energy efficient congestion detection and avoidance in sensor networks," ACM Transactions on Sensor Networks, vol. 7, no. 4, article 32, 2011.

[11] S. Deering and R. Hinden, "Internet protocol, version 6 (IPv6) specification," Tech. Rep. RFC 2460, Internet Engineering Task Force, 1998.

[12] H. A. Al-Kashoash, H. M. Amer, L. Mihaylova, and A. H. Kemp, "Optimization based hybrid congestion alleviation for 6lowpan networks," IEEE Internet of Things Journal, 2017.

[13] C.-Y.Wan, S. B. Eisenman, A. T. Campbell, and J. Crowcroft, "Siphon: overload traffic management using multiradio virtual sinks in sensor networks," in Proceedings of the 3rd ACM International Conference on Embedded Networked Sensor Systems (SenSys '05), pp. 116-129, San Diego, Calif, USA, November 2005.

[14] M. M. Bhuiyan, I. Gondal, and J. Kamruzzaman, "LACAR: location aided congestion aware routing in wireless sensor networks," in Proceedings of the IEEEWireless Communications and Networking Conference (WCNC '10), pp. 1-6, Sydney, Australia, April 2010.

[15] F. Ren, T. He, S. K. Das, and C. Lin, "Traffic-aware dynamic routing to alleviate congestion in wireless sensor networks," IEEE Transactions on Parallel and Distributed Systems, vol. 22, no. 9, pp. 1585-1598, 2011.

[16] D.DebBarma,Q.Wang, I. G. M. M.Niemegeers, S.H. deGroot, and A. Lo, "Coalition game-theory-based congestion control in hybrid FiWi indoor network," in Proceedings of the $3^{\text {rd }}$ International Conference on Future Generation Communication Technologies (FGCT '14), pp. 25-32, August 2014.

[17] C. T. Li, J. K. Wang, B. Wang, and Y. H. Han, "An efficient compressed sensing-based cross-layer congestion control scheme for Wireless Sensor Networks," in Proceedings of the 26th Chinese Control and Decision Conference (CCDC '14), pp.637-641, Changsha, China, June 2014. 